\title{
RAPID SHORT-TERM PULSE-PERIOD VARIATIONS IN VELA X-1 (4U 0900-40)
}

\author{
M. van der Klis**** and J. M. Bonnet-Bidaud*** \\ *Astronomical Institute 'Anton Pannekoek', University \\ of Amsterdam, Roetersstraat 15, $1018 \mathrm{WB}$ Amsterdam, \\ The Netherlands \\ * Cosmic-Ray Working Group, Leiden, The Netherlands \\ ***Section d'Astrophysique, CEN-Saclay, DPh-EP/Ap, F-91191 \\ Gif-sur-Yvette, France
}

\begin{abstract}
Results are presented of an analysis of 83 days of $2-12 \mathrm{keV} X$-ray observations of Vela $X-1$ (4U 0900-40) obtained during three separate pointings with the ESA COS-B satellite. The pulsation period is shown to undergo very rapid intrinsic changes, at a rate of up to $P / P \sim 10^{-1} \mathrm{yr}^{-1}$ during intervals of a few days. The lower values of $\dot{P} / \mathrm{P} \sim 10^{-3} \mathrm{yr}-1$ which were previously observed over longer intervals, appear to result from an averaging-out of these rapid changes. It is argued, that the transfer of angular momentum to the neutron star by the accreting matter is very unlikely to be sufficient to explain these pulsation-period changes. An alternative explanation is suggested.
\end{abstract}

\section{KEYWORDS}

$X$-ray binaries; $X$-ray pulsations; neutron stars; angular-momentum transfer; accretion torques; Vela $X-1 ; 4 U$ 0900-40.

\section{INTRODUCTION}

Vela $X-1$ (4U 0900-40) is a massive $X$-ray binary which contains a neutron star with a rotation period of $\sim 283 \mathrm{~s}$. It is widely believed that accretion is taking place in this source by capture of matter from the strong stellar wind of the optical companion star (Conti, 1978; Dupree et al., 1980).

Between 1974 and 1979 the neutron star has been spinning up at an average rate of $\dot{P} / P \sim$ $-1.510^{-4} \mathrm{yr}^{-1}$. This is compatible with the rate expec ced from simple estimates of the amount of angular momentum transferred to the neutron star by matter accreting from the stellar wind. From 1979 until 1982, however, the pulsation period did increase at an average rate of $\dot{\mathrm{P}} / \mathrm{P} \sim+2.510^{-4} \mathrm{yr}^{-1}$ (Nagase et al., this volume). On shorter time scales (months to weeks) more rapid spin-up and spin-down episodes have been reported, with rates of up to $|P / P| \sim$ $410^{-4} \mathrm{yr}^{-1}$ (Ógelman et al., 1977; Nagase et al., 1982).

Theoretical efforts to understand the pulse-period derivative variations in Vela $X-1$ and similar systems have concentrated on the effects of inhomogeneities in the accreting matter (Wang, 1981; Davies and Pringle, 1980), the effects of disk accretion (Ghosh and Lamb, 1979) and of torques originating in the interior of the neutron star (Lamb et al., 1978). It is not clear whether any of these mechanisms could explain the very rapid pulse period variations presented in this paper, and we suggest an alternative explanation.

For a full account of the COS-B X-ray observations of Vela X-1, see van der Klis (1983) and van der Klis and Bonnet-Bidaud (1983).

\section{OBSERVATIONS}

Vela X-1 was observed with the $2-12 \mathrm{keV} X$-ray detector of the COS-B satellite on three occasions. The total duration of the observations was 83 days. Figure 1 is a plot of the background-subtracted $x$-ray flux as a function of time. The gaps in the data are caused by satellite switch-off during passage of the radiation belts and by occasional solar charged particle storms. The flaring which is visible in Fig. 1 is real (and a well-known phenomenon in Vela $X-1$, e.g. Charles et al., 1978), except in some cases during the last observation, when severe background variability was occurring. In all three observations the expected eclipses of the source are visible between the dashed lines. 


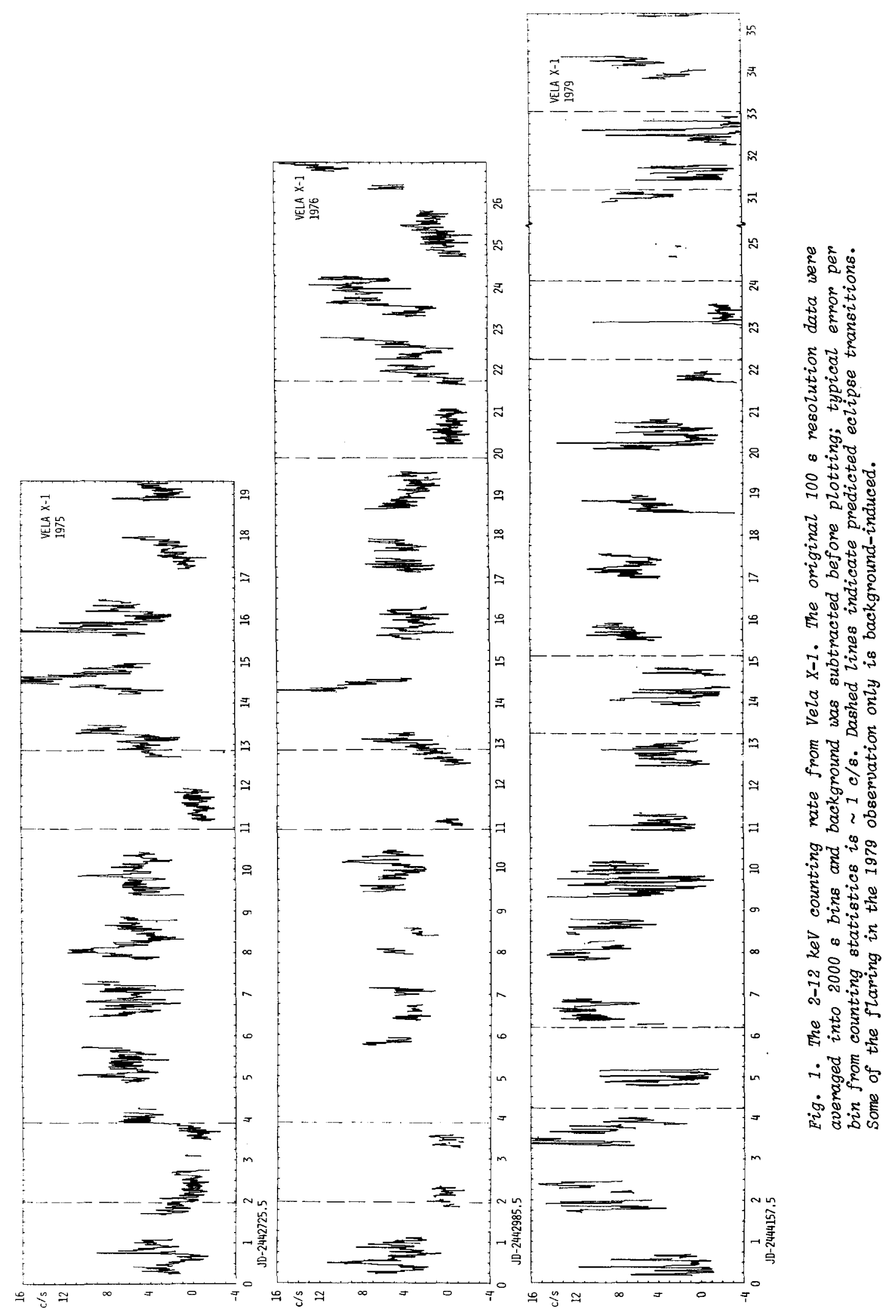




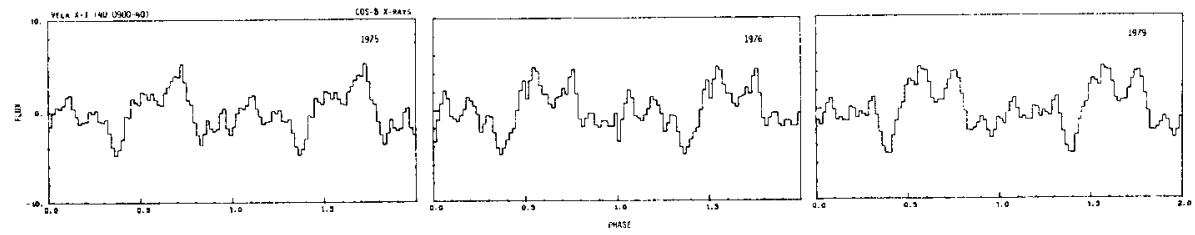

Fig. 2. Average pulse profiles obtained by folding modulo the $\sim 283 s$ pulsation period. Vertical scale is arbitrary. Bin width is $\sim 5 \mathrm{~s}$, statistical error per bin is $\sim 0.8$ units. Profiles are repeated once for clarity.

For the study of the pulsation we used data with a time resolution of $0.25 \mathrm{~ms}$. Figure 2 shows the average pulse profile obtained in each observation by folding these data modulo the pulsation period, after correction for the relative motion of the satellite and the neutron star. The pulse profile has the usual shape in this energy region (McClintock et a1., 1976): a double pulse of which the major pulse is divided into two, and the minor, less clearly, into three sub-pulses, a sharp minimum preceding the major pulse.

\section{ANALYSIS}

To investigate the short-term behaviour of the pulsation period, the data were divided into segments with a duration of typically $0.2-0.3 \mathrm{~d}$. In each segment the average pulse profile was obtained. The arrival time of each individual average pulse profile was found by crosscorrelating it with a template pulse profile. The obtained arrival times show the effects of the orbital motion of the neutron star leffects of earth and satelite motion were removed at the outset of the analysis) and of the intrinsic variations of the pulsation period. We determined the best fit for the orbital parameters of the neutron star, the pulsation period and its derivative, and removed the orbital effect from the arrival times.

The results of this analysis are given in Fig. 3, which shows the intrinsic variations in the arrival time of the pulsation with respect to a constant pulsation-period ephemeris. The dashed lines are parabolic curves; they correspond to the best-fit values of the average pulsationperiod derivative in each of the three observations; $\dot{P} / P \sim 1.6,0.0$ and $1.010^{-3} \mathrm{yr}^{-1}$, respectively. There are clearly significant variations from these best-fit curves, which in the first observation can be seen to behave in a systematic way, high values of $|\dot{P}|$ being attained around days 9,10 and 19 . These deviations are not correlated to orbital phase, and must be due to intrinsic variations in the pulse-arrival time. If they are interpreted as variations in the pulsation period, they correspond to values of the pulsation-period derivative of between -5 and $+10^{-2} \mathrm{yr}^{-1}$ over intervals of 3 to 4 days.

\section{DISCUSSION}

The average pulsation period derived from each of our three observations fits in well with the general long-term trend of the pulsation period of Vela $X-1$ as seen with other experiments (Nagase et al., this volume). Also, the derived average values of the pulsation-period derivative inside each observation are consistent with the values determined with the Hakucho satellite over similar time intervals (Nagase et a1., 1982).
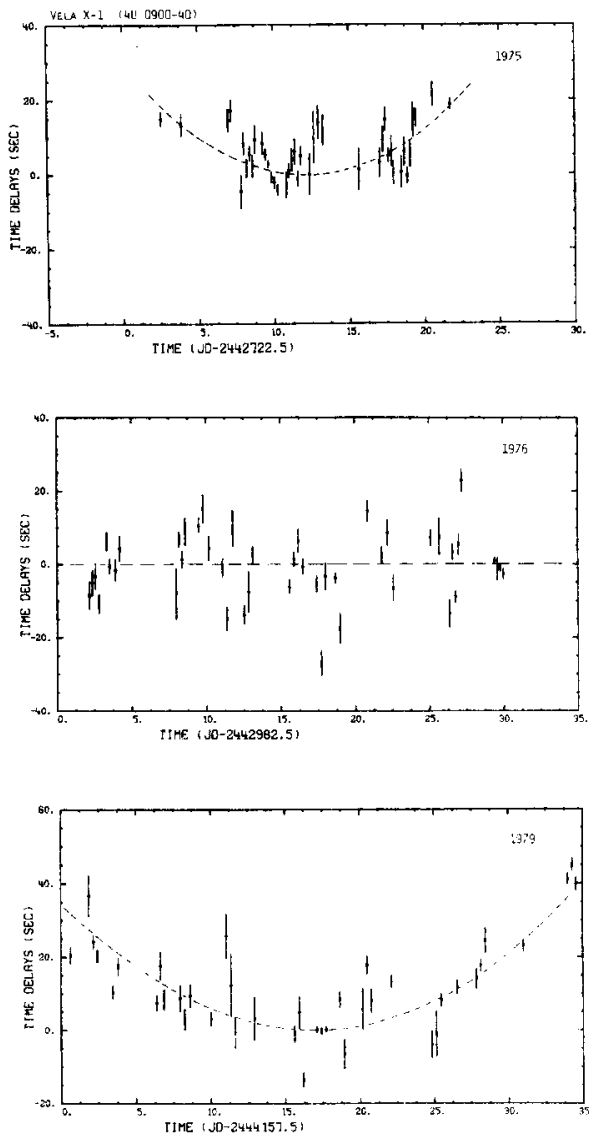

Fig. 3. Pulse arrival times corrected for relative motion between satellite and neutron star. Plotted is the delay with respect to a constant pulse-period ephemeris, with $P=282.92,282.82$ and $282.78 \mathrm{~s}$, respectively. Error bars are $1 \sigma$. Clear deviations with respect to the bestfit parabolic curves are apparent. Note the strong systematic variations of the arrival times during days 8-11 and 18-20 in the first observation. 
Our data suggest, however, that processes acting on a time scale of a few days or less can strongly influence the pulse arrival times. Consequentiy, average pulse period derivatives determined over time windows of the order of 10 days may in some cases rather reflect the window position and the sampling of the data, than any meaningful property of the source.

The question remains what is the cause of the observed rapid pulse arrival-time fluctuations.

We know that changes in the shape of the pulse profile do occur in Vela X-1 (Makishima and Nagase, 1980), and it is clear that in a cross-correlation method as employed here, these might be translated into apparent phase shifts. In our case, each individual pulse profile is the average of $60-90$ pulses, and the $\chi^{2}$-values of the cross-correlations do not indicate that strong shape-changes are occurring. However, shape-changes of the pulse profile can never be excluded as a cause of the arrival-time fluctuations, as these changes might mainiy occur in the form of a phase shift of the profile (not due to a change in the rate of rotation of the neutron star). If, for example, the beaming mechanism which is responsible for the formation of the pulses is connected with the direction of the magnetic field lines, then bending of the field lines by 10 or $20^{\circ}$ could in principle change the direction in which the pulses are emitted sufficiently to explain the observed arrival time fluctuations. Shifts in the position of the hot spot on the neutron star surface could have a similar effect.

Angular momentum transfer by accreting matter is unlikely to be the explanation for the arrival-time fluctuations, mainly for two reasons:

- We observe no correlation between the pulse period derivative and the X-ray luminosity.

- The specific angular momentum which the accreting matter should have in order to explain the arrival-time fluctuations is so large, that at the edge of the magnetosphere the infalling matter would have to move with a transverse velocity which is greatly exceeding the escape velocity, so that accretion would be prevented.

It is anyhow questionable that the matter could obtain the high required angular momentum: estimates of the angular-momentum capture from a spherically symmetric wind lead to the conclusion that this would require a relative velocity between the neutron star and the matter in the wind of $\sim 10^{2} \mathrm{~km} \mathrm{~s}^{-1}$ or less, lower than the orbital velocity and much below the observed wind velocities of $\sim 10^{3} \mathrm{~km} \mathrm{~s}^{-1}$ (Dupree et al., 1980).

On the other hand, small, dense blobs of matter moving through the stellar wind (see, e.g., White et al., 1983) which, because of their high velocity, are not captured by the neutron star, but which do manage to transfer angular momentum through interaction with the magnetic field would in principle conform to the observational constraints. However, in order to carry a sufficient amount of momentum, these blobs should be very massive, $\sim 10^{23} \mathrm{~g}$ at a typical velocity of a few $10^{3} \mathrm{~km} \mathrm{~s}^{-1}$.

In general, explanations for the pulse arrival-time fluctuations in terms of changes in the rate of rotation of the neutron star tend to lead to rather extreme conclusions because of the very large torques which are required. Therefore, an explanation involving changes in the beaming direction as suggested above may be more attractive.

MvdK acknowledges support by ZWO (ASTRON).

\section{REFERENCES}

Davies, R.E., Pringle, J.E., 1980, Monthly Notices Roy. Astr. Soc. 191, 599.

Charles, P.A., Mason, K.0., White, N.E., Culhane, J.L., Sanford, P.W., Moffat, A.F.J., 1978, Monthiy Notices Roy. Astr. Soc. 183, 813.

Conti, P.S., 1978, Astron. Astrophys. 63, 225.

Dupree, A.K. et (19) al., 1980, Astrophys. J. 238, 969.

Ghosh, P., Lamb, F.K., 1979, Astrophys. J. 234, 296.

Lamb, F.K., Pines, D., Shaham, J., 1978, Astrophys. J. 224, 969.

Makishima, K., Nagase, F., 1980, Symp. on Space Astrophys., Tokyo.

McClintock, J.E., Rappaport, S., Joss, P.C., Bradt, H., Buff, J., Clark, G.W., Hearn, D.,

Lewin, W.H.G., Matilsky, T., Mayer, W. Primini, F., 1976, Astrophys. J. 206, L99.

Nagase, F. and Hakucho team, 1982, ISAS RN 178.

Ogelman, H., Beuermann, K.P, Kanbach, G., Mayer-Hasselwander, H.A., Capozzi, D.,

Fiordílino, E., Molteni, D., 1977, Astron. Astrophys. 58, 385.

Van der Klis, M., 1983, Ph. D. thesis, Univ. of Amsterdam.

Van der Klis and Bonnet-Bidaud, 1983, to appear in Astron. Astrophys.

Wang, Y.-M., 1981, Astron. Astrophys, 102, 36.

White, N.E., Kallmann, T.R., Swank, J.H., 1982, Astrophys. J. 269, 264. 